\begin{tabular}{cc} 
Sharif University of Technology \\
Scientia Iranica \\
SCIENTIA & Transactions B: Mechanical Engineering \\
I RAN I CA & \\
\hline
\end{tabular}

\title{
Application of AG method and its improvement to nonlinear damped oscillators
}

\author{
M. Mohammadian ${ }^{a, *}$ and M. Shariati ${ }^{b}$ \\ a. Department of Mechanical Engineering, Kordkuy Center, Gorgan Branch, Islamic Azad University, Kordkuy, P.O. Box \\ 488164-4479, Iran. \\ b. Department of Mechanical Engineering, Faculty of Engineering, Ferdowsi University of Mashhad, Mashhad, P.O. Box \\ 91775-1111, Iran.
}

Received 19 May 2016; received in revised form 18 September 2016; accepted 29 October 2018

\author{
KEYWORDS \\ Nonlinear frequency; \\ Akbari-Ganji's \\ Method (AGM); \\ Variational Iteration \\ Method (VIM); \\ Semi-analytical \\ technique; \\ Damped Duffing \\ oscillator; \\ Flexible beam with \\ damping; \\ Transversal vibration; \\ Quintic nonlinear \\ beam.
}

\begin{abstract}
In this paper, a new and innovative semi-analytical technique, namely AkbariGanji's Method (AGM), is employed for solving three nonlinear damped oscillatory systems. Applying this method to nonlinear problems is very simple because, in the solving process of only a trial solution, the main differential equation and its derivatives are required. The analytical solutions obtained by AGM are utilized to study the impact of amplitude on nonlinear frequency and damping ratio. It is found that AGM produces acceptable results for the problems considered in this paper. Moreover, in order to obtain a more accurate solution, instead of using a trial solution with higher-order terms that may result in complicated and time-consuming mathematical calculations, the solution obtained by AGM is improved via Variational Iteration Method (VIM). The usefulness and effectiveness of the approach is demonstrated through the comparison of the obtained results and those achieved by the numerical method. Hence, AGM can be applied to nonlinear problems consisting of significant nonlinear damping terms and, if necessary, can be easily improved.

(C) 2020 Sharif University of Technology. All rights reserved.
\end{abstract}

\section{Introduction}

Studying nonlinear damped oscillatory systems is an important issue for researchers because there are so many phenomena in structural mechanics and physics that can be modeled like a nonlinear oscillator including strong nonlinearities and damping terms [13]. For example, studying the transverse vibration of a simply supported flexible beam subjected to an axial force leads to a governing nonlinear differential equation containing cubic-quintic nonlinearities and

*. Corresponding author. Tel.: +981732173717 E-mail addresses: mo.mohammadyan@gmail.com ( $M$. Mohammadian); mshariati44@gmail.com (M. Shariati)

doi: $10.24200 /$ sci. 2018.21093 linear and nonlinear damping terms [4]. When an oscillatory system includes damping, there is a nonconservative system and, in contrast to the conservative one [5-8], the amplitude of oscillations decreases over time. Most of analytical approaches such as maxmin approach [9,10], Hamiltonian approach [11], and modified variational technique [12] are unable to handle non-conservative systems.

Baltanás et al. [13] studied the impact of a nonlinear term proportional to the power of velocity and energy dissipation over a cycle in double-well Duffing oscillator. Liao [14] developed the Homotopy Analysis Method to positively damp the vibrations of oscillators, and showed that the method was valid for systems with small or large parameters. Studying oscillators with nonlinear elastic and damping forces was performed by Cveticanin [15]. He derived a 
second-order differential equation for the problem, and showed that the obtained analytical solutions were in good agreement with the numerical results. Shamsul Alam et al. [16] combined the Krylov-BogoliubovMitropolskii (KBM) and harmonic balance methods to obtain approximate solution of damped oscillatory systems. They reported that the combination of extended KBM and harmonic balance methods led to the desired results. In another work, Newton's method and harmonic balance method were combined by Wu and Sun [17] for obtaining the analytical approximate solutions of strongly nonlinear damped oscillators. The proposed method can approach very accurate solutions in a few iterations even if damping and nonlinearities are high in the system. Nourazar and Mirzabeigy [18] applied Differential Transform Method (DTM) to a nonlinear Duffing oscillator with damping effect under different boundary conditions. They showed that the results of DTM were only valid within a small range of time domains. Hence, they improved the results by combining the DTM with Laplace transform and Pade technique. Numerical results confirmed the accuracy of their approach. Cveticanin [19] applied harmonic balance method to study the nonlinear damped-van der Pol oscillator. He obtained a second-order differential equation with nonlinear terms and, also, linear and nonlinear damping terms. The comparison of the results and those obtained numerically confirmed his procedure. An exponential non-viscous damped oscillator was investigated by Ene et al. [20]. They proposed an Optimal Homotopy Perturbation Method to solve the nonlinear oscillator. They showed that their method was very accurate and, thus, the existing small or large parameters in the system were not necessary. In addition, the same method was employed by Herisanu and Marinca [21] and applied to the non-conservative system of a rotational electrical machine. Further to that, Razzak and Molla [22] combined Struble's method with Homotopy Perturbation Method (HPM) and proposed a new analytical approach for obtaining approximate solutions of a nonlinear oscillator with considerable damping effect.

Recently, a new semi-analytical approach, which is called the Akbari-Ganji' Method (AGM), has been proposed for solving nonlinear equations. In this method, a trial solution is assumed for the problem and, then, through a set of algebraic calculations, the constant parameters of trial solution such as angular frequency and phase angle are determined. In comparison with other semi-analytical techniques such as the DTM [23,24], the optimal homotopy perturbation method [25], and the Adomian decomposition method [26], AGM has a very simple solving process such that only the initial conditions, the main differential equation, and its derivatives are required. Hence, the method can be easily implemented even by students with intermediate mathematical knowledge. Furthermore, in contrast to the perturbation method [27], the presence of small parameters is not required. The application of AGM can be found in some strong nonlinear problems such as Duffing, Vanderpol and Rayleigh equations [28,29]. Further, Akbari et al. [30] applied AGM to two complicated nonlinear differential equations. In the first one, they analyzed the structures with the variable cross-section and materials and, in the second problem, they studied one-dimensional heat transfer with various logarithmic surfaces and heat generation. In another work, the nonlinear differential equation governing on a rigid beam on the viscoelastic foundation was investigated by AGM [31].

Besides all the advantages of AGM, in some cases, it may need to be improved. For example, in the solving process of Duffing equation with cubic nonlinearities, Mirgolbabaee et al. [32] assumed a trial function up to three terms to obtain an accurate solution. The same authors in another work [29] supposed a solution up to four terms for large values of amplitude to increase the precision of solution. This treatment using higherorder terms for trial solution led to a set of equations, including seven equations with seven unknowns and nine equations with nine unknowns in [32] and [29], respectively. Solving such a set of equations, which are nonlinear with respect to unknowns, may be sometimes cumbersome or impossible. Moreover, the situation may be more complicated when the quadratic, quintic nonlinearities and nonlinear damping terms exist together in the problem.

In the current paper, AGM is applied to nonconservative oscillators. Three damped systems with strong nonlinearities are considered, and their nonlinear frequency, displacement, and phase plane diagrams are obtained. In addition, the efficiency of AGM is examined for non-zero initial conditions. It is shown that using a trial solution with only one term leads to accurate results for problems considered here. In situations where a more accurate solution is required, contrary to the previously published papers that have utilized a trial solution with higher-order terms, it is shown that AGM can be easily improved by Variational Iteration Method (VIM). Moreover, the effects of amplitude and system parameters on nonlinear frequency and damping ratio are studied. The comparison of the results obtained by AGM and those predicted by the numerical method reveals the correctness and usefulness of the approach.

\section{Basic idea of the AGM}

In general, the nonlinear governing differential equation of a vibrational system can be defined as follows:

$$
f\left(\ddot{u}, \dot{u}, u, F_{0} \sin \left(\omega_{0} t\right)\right)=0,
$$


where $\omega_{0}$ and $F_{0}$ are the angular frequency and amplitude of the external harmonic force, respectively. The initial conditions are as follows:

$$
u(0)=A, \quad \dot{u}(0)=B .
$$

The answer of Eq. (1) is introduced as the sum of the particular solution $\left(u_{p}\right)$ and the harmonic solution $\left(u_{h}\right)$ as follows:

$$
\begin{aligned}
u(t)= & u_{h}+u_{p}=e^{-a t}\{C \cos (\omega t)+D \sin (\omega t)\} \\
& +M \cos \left(\omega_{0} t\right)+N \sin \left(\omega_{0} t\right) .
\end{aligned}
$$

Eq. (3) can be expressed as in the following form:

$$
u(t)=e^{-a t}\{b \cos (\omega t+\varphi)\}+d \cos \left(\omega_{0} t+\Phi\right),
$$

where $b=\sqrt{C^{2}+D^{2}}, d=\sqrt{M^{2}+N^{2}}, \varphi=$ $\arctan (D / C)$, and $\Phi=\arctan (N / M)$. In order to increase the accuracy of Eq. (3), one can add another term in the form of cosine to it. Hence, the exact solution of Eq. (1) will be obtained in the following form:

$$
u(t)=e^{-a t}\left\{\sum_{i=1}^{\infty} b_{i} \cos \left(i \omega t+\varphi_{i}\right)\right\}+d \cos \left(\omega_{0} t+\Phi\right),
$$

where $\left\{a, b_{1}, b_{2}, \cdots, \varphi_{1}, \varphi_{2}, \cdots, \omega, d, \Phi\right\}$ are the coefficients that will be obtained by AGM. If there are $n$ unknowns, $n$ equations are required to determine all unknowns. In AGM, the initial conditions are employed to construct the required equations as in the two following steps:

Step 1. The initial conditions given in Eq. (2) are substituted into Eq. (5). Therefore, we have:

$$
\begin{gathered}
\left\{\sum_{i=1}^{\infty} b_{i} \cos \left(\varphi_{i}\right)\right\}+d \cos (\Phi)=A \\
a\left\{\sum_{i=1}^{\infty} b_{i} \cos \left(\varphi_{i}\right)\right\}+\sum_{i=1}^{\infty} i \omega b_{i} \sin \left(\varphi_{i}\right) \\
+d \omega_{0} \sin (\Phi)=B .
\end{gathered}
$$

Now, there are only two equations and, therefore, $(n-$ 2 ) equations are still required that will be constructed in the next step;

Step 2. In this step, the initial conditions are applied to the main differential equation and its derivatives. After choosing a trial function as the answer of Eq. (1), which is given in its general form by Eq. (5), it is substituted into the main differential equation instead of its dependent variable $u$. Considering the trial function as $g(t)$ and substituting it into Eq. (1) instead of $u$, we have:

$$
f(t)=f\left(g^{\prime \prime}(t), g^{\prime}(t), g(t), F_{0} \sin \left(\omega_{0} t\right)\right) .
$$

Now, the initial conditions are substituted into Eq. (8) and its derivatives as follows:

$$
\begin{aligned}
& f(\mathrm{IC})=f\left(g^{\prime \prime}(\mathrm{IC}), g^{\prime}(\mathrm{IC}), g(\mathrm{IC}), \cdots\right), \\
& f^{\prime}(\mathrm{IC})=f\left(g^{\prime \prime}(\mathrm{IC}), g^{\prime}(\mathrm{IC}), g(\mathrm{IC}), \cdots\right), \\
& f^{\prime \prime}(\mathrm{IC})=f\left(g^{\prime \prime}(\mathrm{IC}), g^{\prime}(\mathrm{IC}), g(\mathrm{IC}), \cdots\right),
\end{aligned}
$$

$\cdots$

The higher-order derivatives of Eq. (1) must be applied until the number of yielded equations in Eq. (9) is equal to $(n-2)$. According to Eqs. (6), (7), and (9), a set of algebraic equations including $n$ equations and $n$ unknowns is created. By solving these equations simultaneously, the unknowns are determined.

For a vibrational system without any harmonic external force, the simplest form of trial function regarding Eq. (5) is as follows:

$$
u(t)=e^{-a t}\{b \cos (\omega t+\varphi)\}
$$

where $a, b, \omega$, and $\varphi$ are the unknowns that should be determined. Considering the initial conditions as Eq. (2) and regarding Eqs. (6), (7), and (9), the required equations for obtaining the aforementioned unknowns are summarized as follows:

$$
\begin{aligned}
& b \cos (\varphi)=A, \\
& a b \cos (\varphi)+\omega b \sin (\varphi)=B, \\
& f(\mathrm{IC})=f\left(u^{\prime \prime}(\mathrm{IC}), u^{\prime}(\mathrm{IC}), u(\mathrm{IC})\right), \\
& f^{\prime}(\mathrm{IC})=f\left(u^{\prime \prime}(\mathrm{IC}), u^{\prime}(\mathrm{IC}), u(\mathrm{IC})\right) .
\end{aligned}
$$

Therefore, if the trial function is considered as Eq. (10), only four equations are required to obtain the unknowns. To increase the precision of the obtained solution, one can choose a trial function with higherorder terms, resulting in more unknowns. However, as expressed in the introduction section, this treatment is sometimes conducted on a set of algebraic equations with a cumbersome and even impossible solving process. Hence, in the current paper, the VIM is employed to improve the solution obtained by AGM. In the following section, the basic idea of the VIM is briefly explained.

\section{Application of the VIM to damped oscillatory systems}

The variational iteration method, which was first proposed by $\mathrm{He}$ in 1997 [33], introduces a powerful approximate analytical technique for solving nonlinear differential equations. The main characteristic of this method is to create a correction functional using a general Lagrange multiplier. In order to illustrate 
the main concept of this method, a general nonlinear differential equation is considered as follows:

$$
L[u(t)]+N[u(t)]=g(t),
$$

where $L$ and $N$ are the linear and nonlinear operators, respectively. Moreover, $g(t)$ is an inhomogeneous term. According to the VIM, the correction functional is as follows $[34,35]$ :

$$
\begin{aligned}
u_{n+1}(t)= & u_{n}(t)+\int_{0}^{t} \lambda(\tau)\left\{L\left(u_{n}(\tau)\right)+N\left(\tilde{u}_{n}(\tau)\right)\right. \\
& -g(\tau)\} d \tau
\end{aligned}
$$

where $\lambda$ is the Lagrange multiplier that can be determined optimally through the variational method. In addition, $u_{n}$ is the $n$th approximate solution and $\tilde{u}_{n}$ is considered as a restricted variation, i.e., $\delta \tilde{u}_{n}=0$. For a damped system, Eq. (12) can be rewritten as follows:

$$
m \ddot{u}+c \dot{u}+k u+N[u(t)]=g(t) .
$$

Substituting Eq. (14) into Eq. (13) and, then, calculating the variation of the governed equation, we have:

$$
\begin{aligned}
\delta u_{n+1}(t) & =\delta u_{n}(t)+\delta \int_{0}^{t} \lambda(\tau)\left\{m u^{\prime \prime}+c u^{\prime}+k u\right. \\
& \left.+N\left(\tilde{u}_{n}(\tau)\right)-g(\tau)\right\} d \tau=\delta u_{n}(t) \\
& +\left.\left(-m \lambda^{\prime}+c \lambda\right) \delta u_{n}(\tau)\right|_{\tau=0} ^{t}+\left.m \lambda \delta u_{n}^{\prime}(\tau)\right|_{\tau=0} ^{t} \\
& +\int_{0}^{t}\left(m \lambda^{\prime \prime}-c \lambda^{\prime}+k \lambda\right) \delta u_{n}(\tau) d \tau=0 .
\end{aligned}
$$

Since the values of $u$ and $\dot{u}$ are specific in $t=0$, their variations are equal to zero, i.e., $\delta u_{n}(0)=\delta u_{n}^{\prime}(0)=0$. Hence, the stationary conditions of Eq. (15) result in the following equations:

$$
\begin{aligned}
& m \lambda^{\prime \prime}(\tau)-c \lambda^{\prime}(\tau)+k \lambda(\tau)=0, \\
& 1-m \lambda^{\prime}(t)+c \lambda(t)=0, \\
& \lambda(t)=0 .
\end{aligned}
$$

By solving Eq. (16), the Lagrange multiplier can be determined as in the following form:

$$
\lambda(\tau)=\frac{\sin (\beta(\tau-t))}{m \beta} e^{\frac{c}{2 m}(\tau-t)}
$$

where:

$$
\beta=\frac{\sqrt{4 m k-c^{2}}}{2 m} .
$$

By using the correction functional and the Lagrange multiplier given in Eqs. (13) and (17), respectively, the solution of AGM can be improved. In the following, the procedure with three examples is illustrated.

\section{Illustrative examples}

In this section, three examples are presented to illustrate the usefulness and effectiveness of the current approach.

Example 1. Consider the damped Duffing oscillator as follows:

$$
f(t)=\ddot{u}(t)+\eta \dot{u}(t)+\beta u(t)+\gamma u^{3}(t)=0 .
$$

As explained in Section 2, the answer of Eq. (19) is considered as follows:

$$
\begin{aligned}
& u(t)=e^{-a t}\{b \cos (\omega t+\varphi)\}, \\
& u(0)=A, \quad \dot{u}(0)=0 .
\end{aligned}
$$

According to Step 1 in Section 2, the initial conditions are applied to Eq. (20) as follows:

$$
\begin{aligned}
& u(0)=A \rightarrow b \cos \varphi=A, \\
& \dot{u}(0)=0 \rightarrow a b \cos \varphi+\omega b \sin \varphi=0 .
\end{aligned}
$$

After substituting Eq. (20) into Eq. (19), according to Step 2 in Section 2, the initial conditions are applied to the main differential equation (i.e., Eq. (19)) and its derivative as follows:

$$
\begin{aligned}
f(0) \rightarrow & a^{2} b \cos \varphi+2 a b \omega \sin \varphi-\omega^{2} b \cos \varphi \\
& +\beta b \cos \varphi+\eta(-a b \cos \varphi-b \omega \sin \varphi) \\
& +\gamma b^{3} \cos ^{3} \varphi=0, \\
f^{\prime}(0) \rightarrow & -a^{3} b \cos \varphi-3 a^{2} b \omega \sin \varphi+3 a \omega^{2} b \cos \varphi \\
& +b \omega^{3} \sin \varphi-\beta a b \cos \varphi-\beta b \omega \sin \varphi \\
& +\eta\left(a^{2} b \cos \varphi+2 a b \omega \sin \varphi-\omega^{2} b \cos \varphi\right) \\
& -3 \gamma a b^{3} \cos ^{3} \varphi-3 \gamma \omega b^{3} \cos ^{2} \varphi \sin \varphi=0 .
\end{aligned}
$$

By solving Eqs. (21)-(24), the constant coefficients of Eq. (20) are obtained as follows:

$$
\begin{aligned}
& a=\frac{1}{2} \eta, \quad b=A \sqrt{\frac{4 \beta+4 A^{2} \gamma}{4 \beta+4 A^{2} \gamma-\eta^{2}}}, \\
& \omega=\frac{1}{2} \sqrt{4 \beta+4 A^{2} \gamma-\eta^{2}}, \quad \varphi=-\arctan \left(\frac{a}{\omega}\right) .
\end{aligned}
$$

By substituting the above constants into Eq. (20), AGM solution is obtained as follows: 


$$
\begin{aligned}
u(t)= & e^{-0.5 \eta t}\left\{A \sqrt{\frac{4 \beta+4 A^{2} \gamma}{4 \beta+4 A^{2} \gamma-\eta^{2}}}\right. \\
& \cos \left(\frac{1}{2} \sqrt{4 \beta+4 A^{2} \gamma-\eta^{2}} t\right. \\
& \left.\left.-\tan ^{-1}\left(\frac{\eta}{\sqrt{4 \beta+4 A^{2} \gamma-\eta^{2}}}\right)\right)\right\} .
\end{aligned}
$$

Considering the physical values for Eq. (19) as $\eta=1$, $\beta=20, \gamma=2$, the solution for $A=0.6$ is depicted in Figure 1 and compared by the numerical solution. Good agreement can be seen between the two methods.

The term $e^{-\xi \omega t}$ in a vibrational differential equation is the main factor for damping. In Eq. (20), the term $e^{-a t}$ is the damping factor and, therefore, damping ratio $(\xi)$ can be found as follows:

$$
e^{-\xi \omega t} \equiv e^{-a t} \rightarrow \xi=\frac{a}{\omega}
$$

Hence, for the current problem, we have:

$$
\xi=\frac{\eta}{\sqrt{4 \beta+4 A^{2} \gamma-\eta^{2} t}} .
$$

Using the analytical expressions obtained through Eqs. (25) and (28), one can investigate the influence of system physical parameters on the frequency and damping ratio. For example, Figures 2 and 3 illustrate the variation of frequency and damping ratio with respect to the amplitude for the mentioned physical parameters, respectively. Further, by increasing the amplitude, the nonlinear frequency and damping ratio increase and decrease, respectively.

The investigations showed that for $A>1$ and the same coefficients as those mentioned before, the

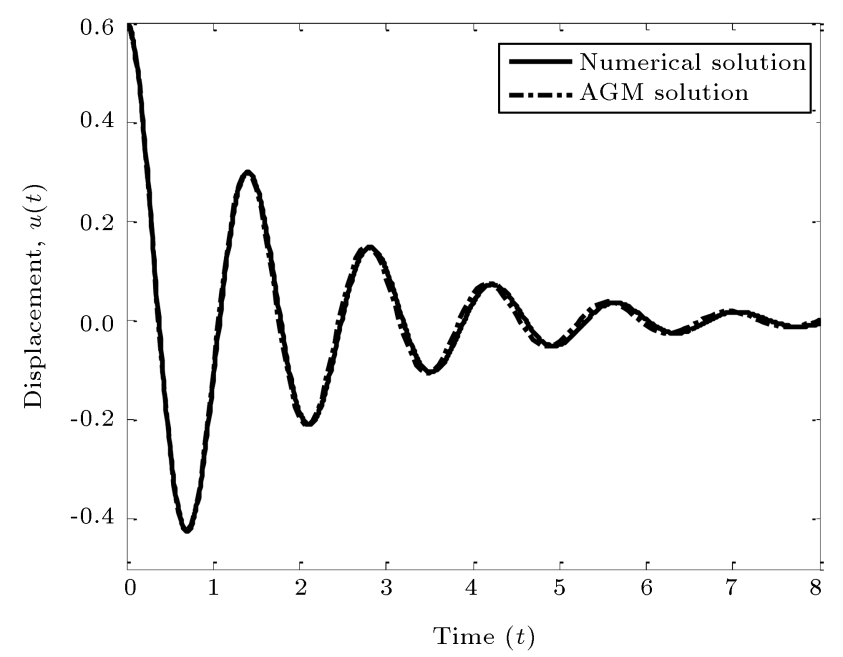

Figure 1. The solutions obtained by Akbari-Ganji's Method (AGM) and numerical method for Example 1, $A=0.6$.

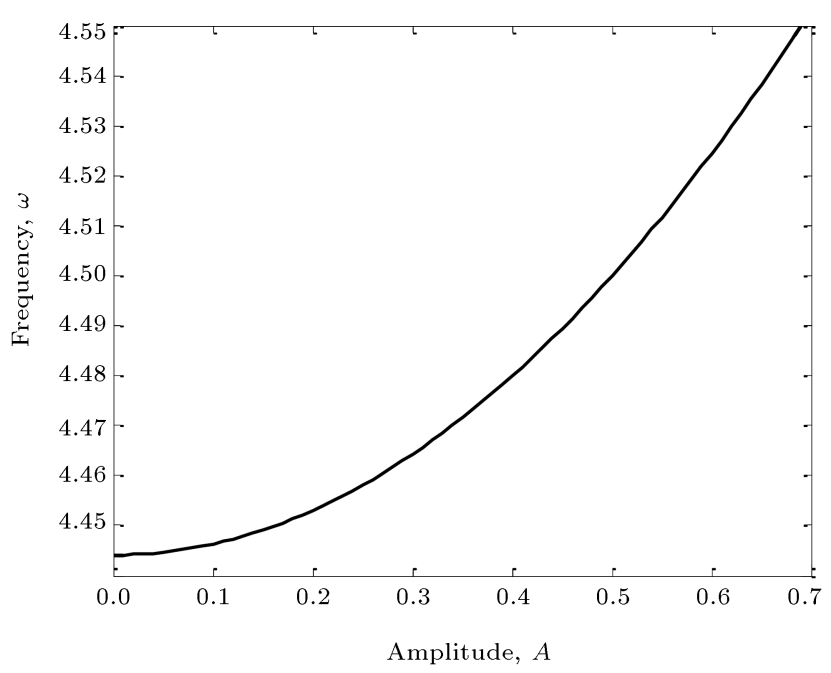

Figure 2. The variation of frequency with respect to the initial amplitude for Example 1.

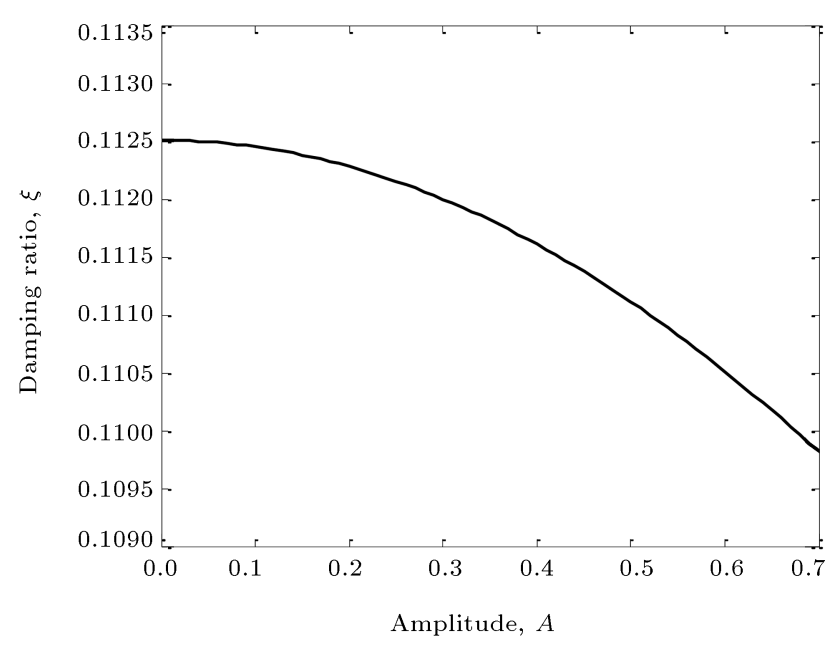

Figure 3. The variation of damping ratio with respect to the initial amplitude for Example 1.

obtained solution in Eq. (26) is of low accuracy. For instance, if the amplitude is selected as $A=1.5$, the following solution will be obtained by AGM:

$$
u(t)=1.5077 e^{-0.5 t} \cos (4.92443 t-0.10119) .
$$

Figure 4 illustrates the above solution and indicates that it requires to be improved. In the current paper, the VIM is employed to improve AGM solution. For this problem, we have $m=c=1$ and $k=20$. After determining $\beta$ through Eq. (18), the Lagrange multiplier is obtained by Eq. (17) as follows:

$$
\lambda(\tau)=\frac{\sin (4.4441(\tau-t))}{4.4441} e^{0.5(\tau-t)} .
$$

By considering Eq. (29) as the zero-order solution $\left(u_{0}(t)\right)$ and substituting it into Eq. (13), the improved solution $\left(u_{\mathrm{IAGM}}\right)$ can be obtained through the following correction functional: 


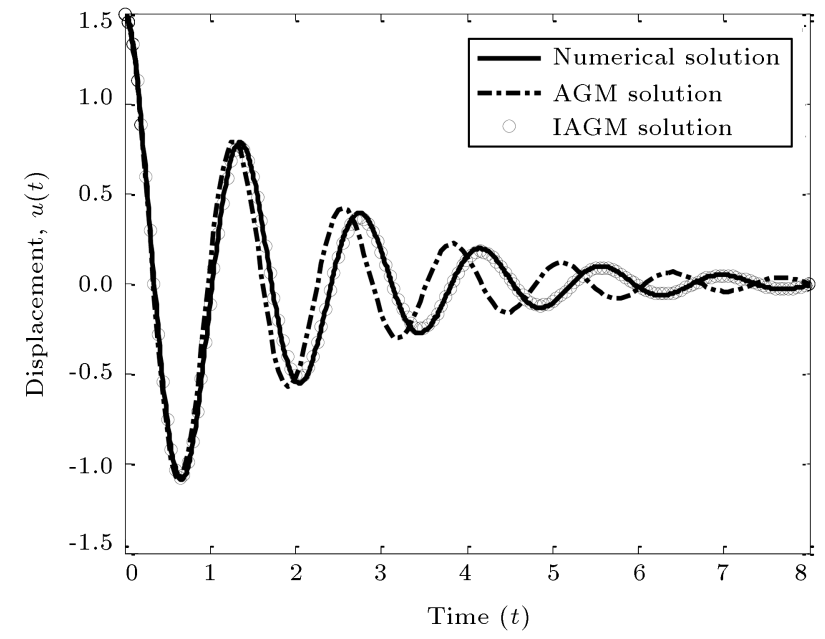

Figure 4. The solutions obtained by Akbari-Ganji's Method (AGM), Improved AGM (IAGM), and numerical method for Example 1, $A=1.5$.

$$
\begin{gathered}
u_{\mathrm{IAGM}}(t)=u_{0}(t)+\int_{0}^{t} \frac{\sin (4.4441(\tau-t))}{4.4441} e^{0.5(\tau-t)} \\
\left\{u_{0}^{\prime \prime}(\tau)+\eta u_{0}^{\prime}(\tau)+\beta u_{0}(\tau)+\gamma u_{0}^{3}(\tau)\right\} d \tau
\end{gathered}
$$

The solution and the phase plane diagrams of the current problem for $A=1.5$ are illustrated in Figures 4 and 5 , respectively. The improved solution obtained by Eq. (31) is in good agreement with the corresponding numerical solution. It should be noted that the phase plane diagram illustrates the oscillator displacement with respect to its velocity. For a conservative system, there is an equilibrium point in which the oscillator has a periodic motion around it. However, for a nonconservative system, the oscillation starts based on the assumed initial conditions and, then, the total

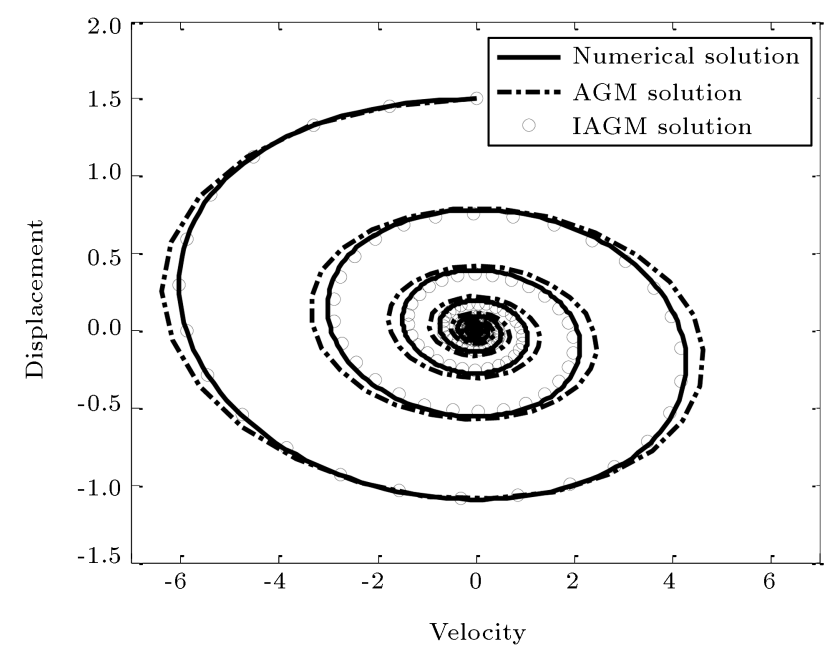

Figure 5. The phase plane diagram obtained by Akbari-Ganji's Method (AGM), Improved AGM (IAGM), and numerical method for Example 1, $A=1.5$. energy of the system gradually decreases due to existing damping in the system. As can be seen, in the phase plane diagram (see Figure 5), the velocity of the system gradually decreases and, eventually, the oscillator comes to rest.

In the following, to investigate the efficiency of AGM with respect to the non-zero initial velocity, the initial conditions are assumed as $u(0)=-0.2$ and $\dot{u}(0)=2$. After solving Eq. (11) for the mentioned system parameters, the following solution is achieved:

$$
u(t)=-0.46959 e^{-0.50727 t} \cos (4.46856 t+1.130834) .
$$

The above solution and its phase plane diagram are depicted in Figures 6 and 7, respectively. As can be seen, there is good agreement between the solution achieved by AGM and the numerical results. For the current problem and the same physical parameters, Nourazar and Mirzabeigy [18] obtained the following solution by the modified differential transform method:

$$
\begin{aligned}
u(t)= & e^{-2.0169 t}(0.00045 \cos (12.6572 t) \\
& +0.0009614 \sin (12.6572 t) \\
& +e^{-0.4965 t}(-0.20045 \cos (4.4826 t) \\
& +0.4239 \sin (4.4826 t)) .
\end{aligned}
$$

The comparison of the two solutions achieved through Eqs. (32) and (33) indicates that AGM solution is more concise.

Example 2. Consider the differential equation of a quadratic nonlinear damped oscillator as follows:

$$
f(t)=\ddot{u}(t)+u(t)+\eta u^{2}(t)+\beta(\gamma+u(t)) \dot{u}(t)=0 .
$$

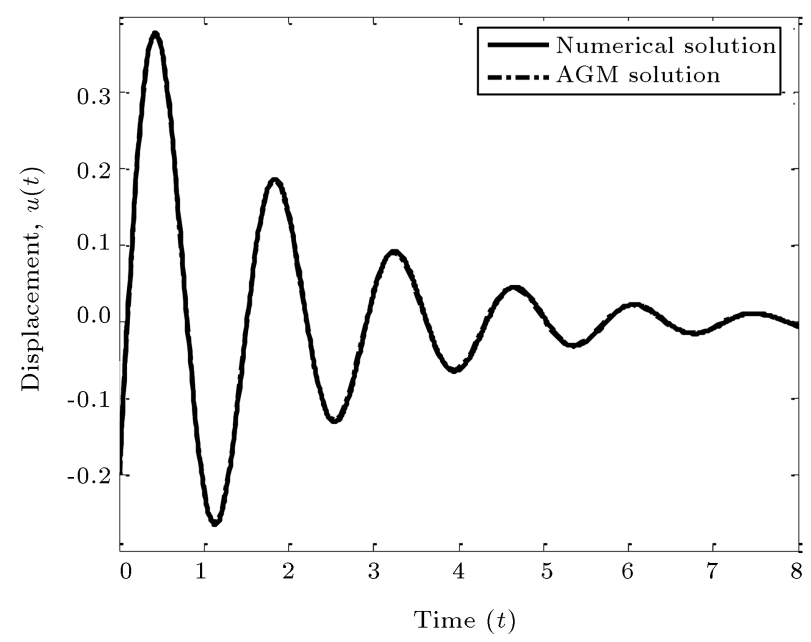

Figure 6. The solutions obtained by Akbari-Ganji's Method (AGM) and numerical method for Example 1, $A=-0.2, \dot{u}(0)=2$. 


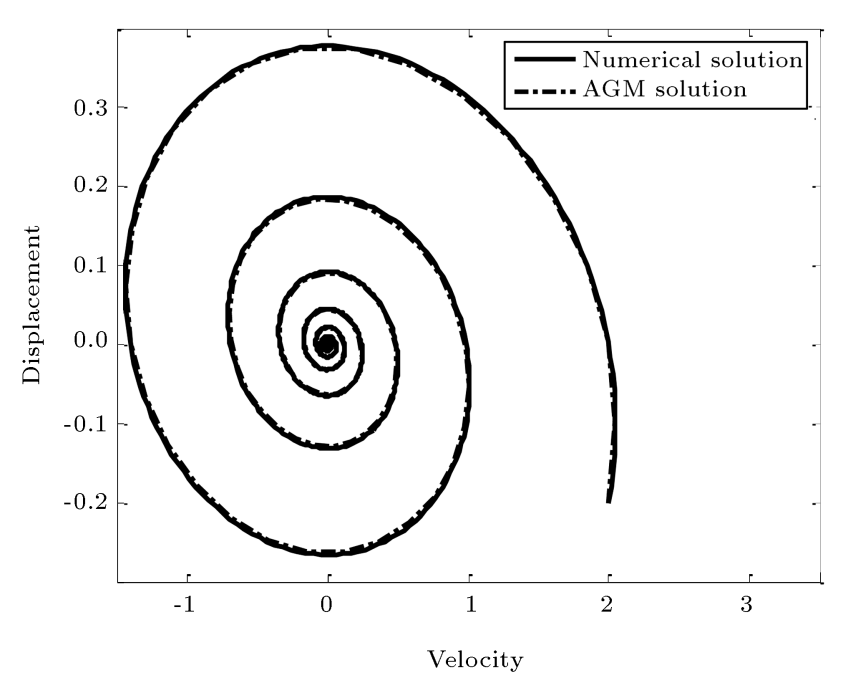

Figure 7. The phase plane diagram obtained by Akbari-Ganji's Method (AGM) and numerical method for Example 1, $A=-0.2, \dot{u}(0)=2$.

Eq. (34) that can present a kinetic model of abrin binding in an Epstein-Barr virus-transformed lymphocyte culture [36] is solved by AGM. In this example, a non-zero value is supposed for the initial velocity. Therefore, the initial conditions are characterized by $u(0)=A, \dot{u}(0)=B$. Considering Eq. (10) as the trial solution of Eq. (34) and regarding the two steps given in Section 2, we have:

$$
\begin{aligned}
u(0)= & A \rightarrow b \cos \varphi=A \\
\dot{u}(0)= & B \rightarrow a b \cos \varphi+\omega b \sin \varphi=B \\
f(0) \rightarrow & a^{2} b \cos \varphi+2 a b \omega \sin \varphi-\omega^{2} b \cos \varphi \\
& +b \cos \varphi+\beta(\gamma+b \cos \varphi)(-a b \cos \varphi-b \omega \sin \varphi) \\
& +\eta b^{2} \cos ^{2} \varphi=0, \\
f^{\prime}(0) \rightarrow & -a^{3} b \cos \varphi-3 a^{2} b \omega \sin \varphi+3 a \omega^{2} b \cos \varphi \\
& +b \omega^{3} \sin \varphi-a b \cos \varphi-b \omega \sin \varphi \\
& -2 \eta b^{2} a \cos { }^{2} \varphi-2 \eta \omega b^{2} \cos \varphi \sin \varphi \\
& +\beta\left(-a b \cos ^{2}-b \omega \sin \varphi\right)^{2}+\beta(\gamma+b \cos \varphi) \\
& \left(a^{2} b \cos \varphi+2 a b \omega \sin \varphi-b \omega^{2} \cos \varphi\right)=0 .
\end{aligned}
$$

Considering the physical values of Eq. (34) as $A=0.1$, $B=0.05, \eta=1.1, \gamma=0.7$, and $\beta=1.2$, the solution to Eq. (34) is determined as follows:

$$
u(t)=0.1411 e^{-0.4569 t} \cos (0.961425 t-0.78304) .
$$

The solution and phase plane diagram obtained by AGM are illustrated in Figures 8 and 9, respectively.

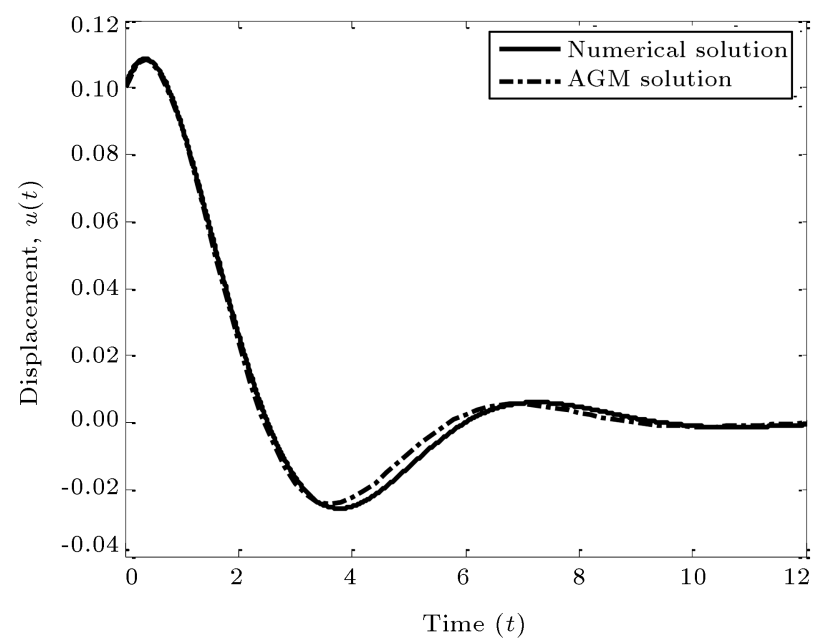

Figure 8. The solutions obtained by Akbari-Ganji's Method (AGM) and numerical method for Example 2, $A=0.1, \dot{u}(0)=0.05$.

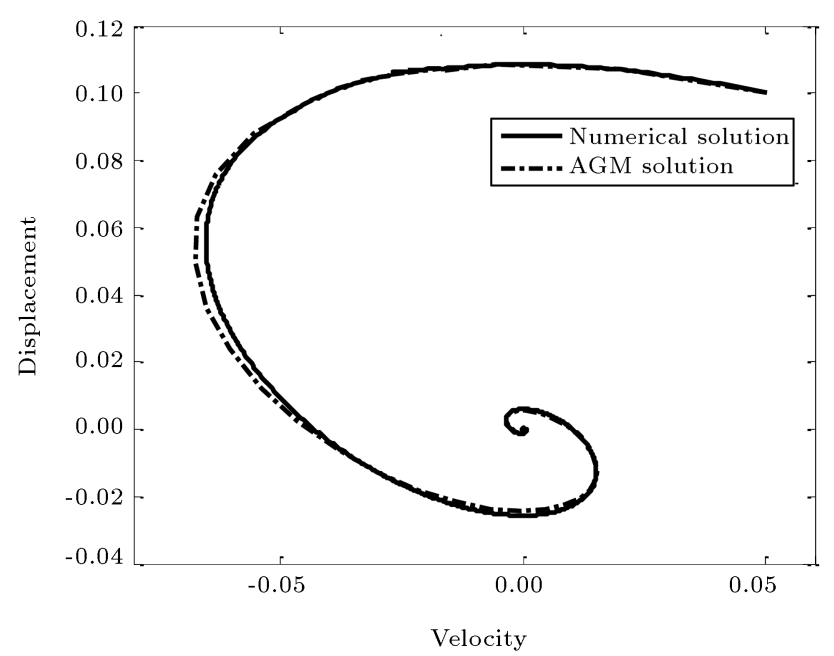

Figure 9. The phase plane diagram obtained by Akbari-Ganji's Method (AGM) and numerical method for Example 2, $A=0.1, \dot{u}(0)=0.05$.

The comparison of AGM and numerical method reveals the high accuracy of AGM solution. Considering the current problem with such parameters as $A=0.5$, $B=0, \eta=1, \gamma=0.54$, and $\beta=1.435$, AGM produces the following solution:

$$
u(t)=0.6305 e^{-0.7462 t} \cos (0.9712 t-0.6551) .
$$

As is depicted in Figure 10, the above solution has low accuracy. Similar to Example 1, the solution obtained by AGM is improved by the VIM. For the current problem, the Lagrange multiplier is as follows:

$$
\lambda(\tau)=\frac{\sin (0.9219(\tau-t))}{0.9219} e^{0.3875(\tau-t)} .
$$

Considering Eq. (40) as the zero-order solution $\left(u_{0}(t)\right)$ and substituting it into Eq. (13), the improved solution 


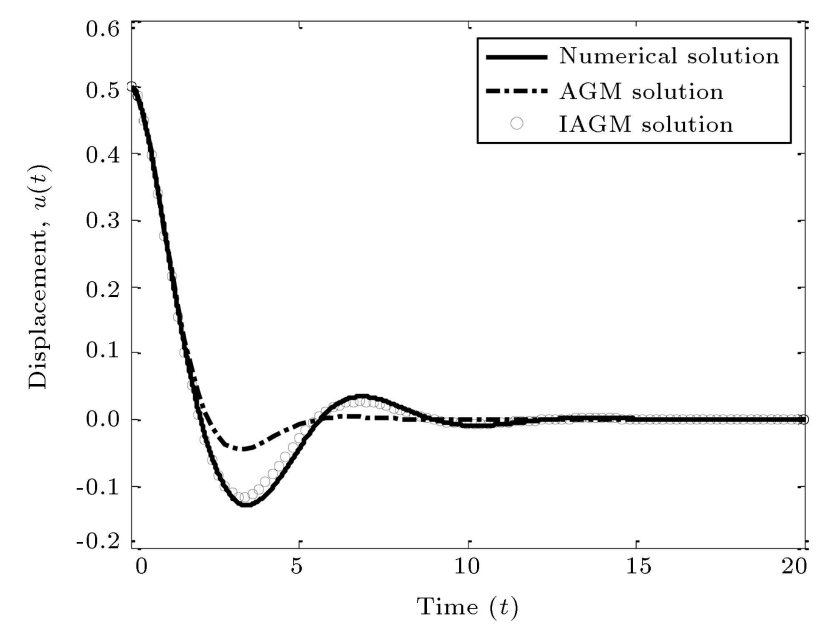

Figure 10. The solutions obtained by Akbari-Ganji's Method (AGM), Improved AGM (IAGM), and numerical method for Example 2, $A=0.5, \dot{u}(0)=0$.

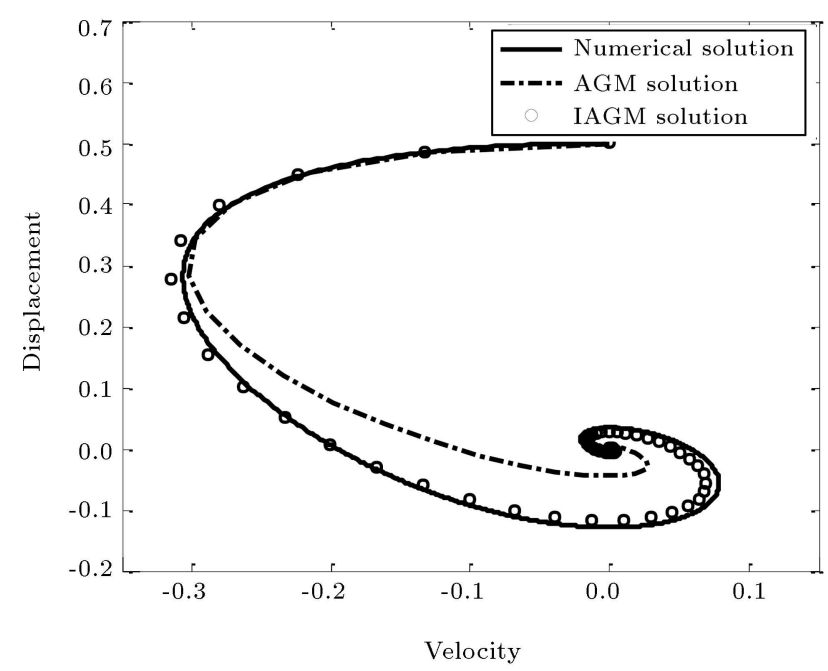

Figure 11. The phase plane diagram obtained by Akbari-Ganji's Method (AGM), Improved AGM (IAGM), and numerical method for Example 2, $A=0.5, \dot{u}(0)=0$.

$\left(u_{\mathrm{IAGM}}\right)$ can be obtained from the following correction functional:

$$
\begin{gathered}
u_{\mathrm{IAGM}}(t)=u_{0}(t)+\int_{0}^{t} \frac{\sin (0.9219(\tau-t))}{0.9219} e^{0.3875(\tau-t)} \\
\left\{u_{0}^{\prime \prime}(\tau)+\beta\left(\gamma+u_{0}(\tau)\right) u_{0}^{\prime}(\tau)+u_{0}(\tau)+\eta u_{0}^{2}(\tau)\right\} d \tau
\end{gathered}
$$

Figures 10 and 11, which show the comparison between the achieved solutions and the numerical method, reveal that the solution of AGM can be easily improved by the VIM.

Example 3. For the last example, the transversal vibration of a flexible beam with simply supported ends subjected to a constant axial load is considered. The governed nonlinear differential equation including nonlinear terms in the damping coefficient is as follows [4]:

$$
\begin{aligned}
f(t)= & \ddot{u}(t)+\left(\mu-\eta u^{2}(t)+\gamma u^{4}(t)\right) \dot{u}+\Omega^{2} u(t) \\
& -\beta u^{3}(t)-\varepsilon u^{5}(t)=0 .
\end{aligned}
$$

The initial conditions are supposed as $u(0)=A$, $\dot{u}(0)=0$. To solve Eq. (43) via AGM, similar to the previous examples, Eq. (10) is supposed as the solution. According to Eq. (11), four equations with four unknowns are constructed as follows:

$$
\begin{gathered}
u(0)=A \rightarrow b \cos \varphi=A \\
\dot{u}(0)=0 \rightarrow a b \cos \varphi+\omega b \sin \varphi=0 \\
f(0) \rightarrow a^{2} b \cos \varphi+2 a b \omega \sin \varphi-\omega^{2} b \cos \varphi+(\mu \\
\left.-\eta b^{2} \cos ^{2} \varphi+\gamma b^{4} \cos ^{4} \varphi\right)(-a b \cos \varphi-b \omega \sin \varphi) \\
\Omega^{2} b \cos \varphi-\beta b^{3} \cos ^{3} \varphi-\varepsilon b^{5} \cos ^{5} \varphi=0 \\
f^{\prime}(0) \rightarrow-a^{3} b \cos \varphi-3 a^{2} b \omega \sin \varphi+3 a \omega^{2} b \cos \varphi \\
+b \omega^{3} \sin \varphi+\left(2 \eta b^{2} a \cos ^{2} \varphi+2 \eta \omega b^{2} \cos \varphi \sin \varphi\right. \\
\left.-4 \gamma a b^{4} \cos { }^{4} \varphi-4 \gamma b^{4} \omega \cos ^{3} \varphi \sin \varphi\right)(-a b \cos \varphi \\
-b \omega \sin \varphi)+\left(\mu-\eta b^{2} \cos ^{2} \varphi+\gamma b^{4} \cos { }^{4} \varphi\right)\left(a^{2} b \cos \varphi\right. \\
\left.+2 a b \omega \sin \varphi-b \omega^{2} \cos ^{2}\right)-\Omega^{2} a b \cos \varphi \\
-\Omega^{2} \omega b \sin \varphi+3 \beta b^{3} a \cos ^{3} \varphi+3 \beta b^{3} \omega \cos { }^{3} \varphi \sin \varphi \\
+5 \varepsilon b^{5} a \cos { }^{5} \varphi+5 \omega \varepsilon b^{5} \cos ^{4} \varphi \sin \varphi=0
\end{gathered}
$$

Solving Eqs. (44)-(47), the constant coefficients of Eq. (10) are obtained as follows:

$$
\begin{aligned}
a & =\frac{1}{2}\left(A^{4} \gamma-A^{2} \eta+\mu\right), & b & =A \sqrt{\frac{\Delta_{1}}{\Delta_{1}+\Delta_{2}}}, \\
\omega & =\frac{1}{2} \sqrt{\Delta_{1}+\Delta_{2}}, & \varphi & =-\arctan \left(\frac{a}{\omega}\right),
\end{aligned}
$$

where:

$$
\begin{aligned}
& \Delta_{1}=-4 A^{2} \beta+4 \Omega^{2}-4 A^{4} \varepsilon \\
& \Delta_{2}=-A^{8} \gamma^{2}+2 A^{6} \gamma \eta-2 A^{4} \gamma \mu-A^{4} \eta^{2}+2 A^{2} \eta \mu-\mu^{2} .
\end{aligned}
$$

Hence, the solution to Eq. (43) is as follows:

$$
\begin{aligned}
u(t)= & e^{-0.5\left(A^{4} \gamma-A^{2} \eta+\mu\right) t}\left\{A \sqrt{\frac{\Delta_{1}}{\Delta_{1}+\Delta_{2}}} \cos \right. \\
& \left.\left(\frac{1}{2} \sqrt{\Delta_{1}+\Delta_{2}} t-\tan ^{-1}\left(\frac{A^{4} \gamma-A^{2} \eta+\mu}{\sqrt{\Delta_{1}+\Delta_{2}}}\right)\right)\right\} .
\end{aligned}
$$

Considering the physical values of Eq. (50) as $\mu=2$, 
$\eta=1.5, \gamma=2.5, \Omega=8, \beta=3.5$, and $\varepsilon=2$, the displacement and phase plane diagrams for $A=0.5$ are depicted and compared by numerical solution in Figures 12 and 13, respectively. Good agreement between the two methods can be seen. According to Eq. (27), the damping ratio $(\xi)$ for the current problem can be found as follows:

$$
\xi=\frac{A^{4} \gamma-A^{2} \eta+\mu}{\sqrt{\Delta_{1}+\Delta_{2}}},
$$

where $\Delta_{1}$ and $\Delta_{2}$ are given in Eq. (49). Eqs. (48) and (51) can be utilized for studying the influence of system physical parameters on the frequency and damping ratio. For example, the variation of frequency and damping ratio with respect to the amplitude for the mentioned physical parameters under different values of $\mu$ are illustrated in Figures 14 and 15, respectively.

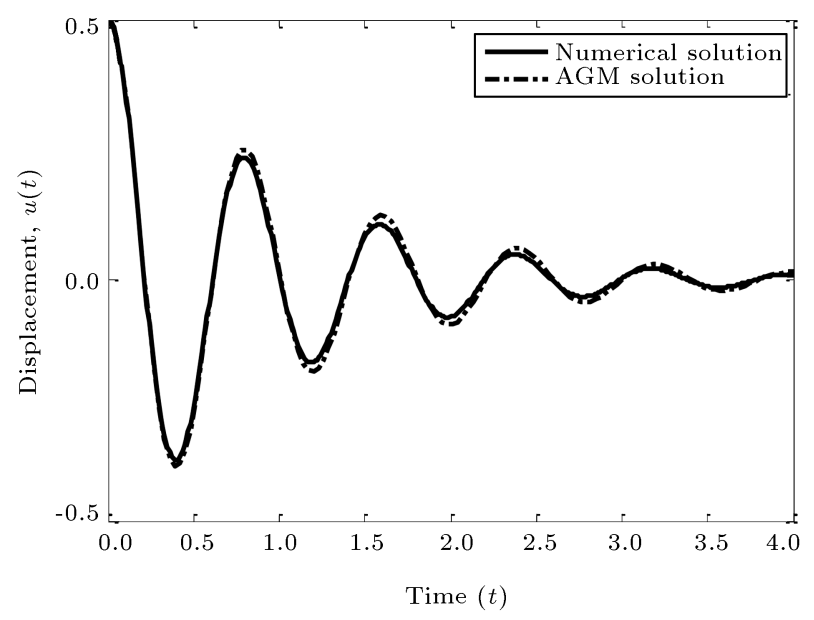

Figure 12. The solutions obtained by Akbari-Ganji's Method (AGM) and numerical method for Example 3, $A=0.5$.

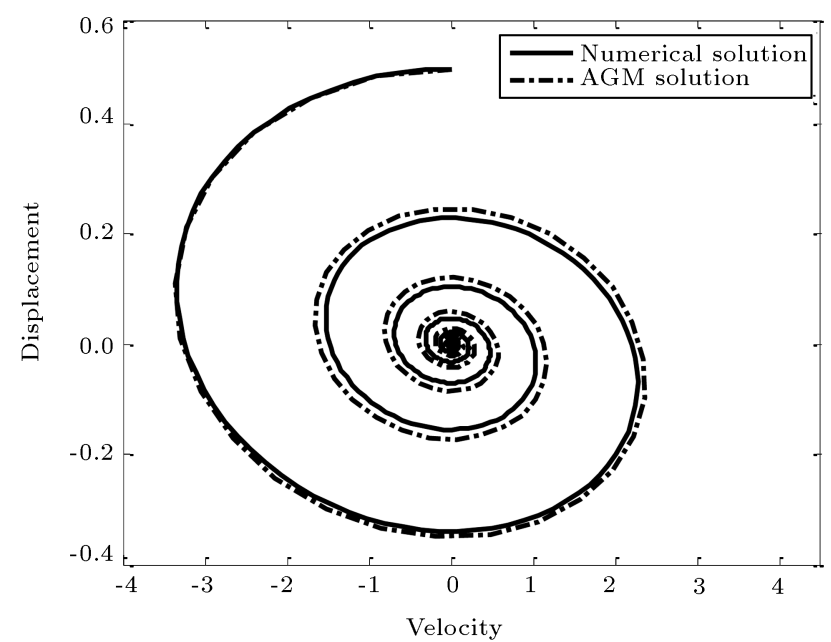

Figure 13. The phase plane diagram obtained by Akbari-Ganji's Method (AGM) and numerical method for Example 3, $A=0.5$.
Figure 14 reveals that, by increasing the amplitude, the nonlinear frequency decreases. In addition, for a specific value of amplitude, increasing $\mu$ results in a smaller frequency. Regarding Figure 15, it can be seen that, by increasing the amplitude, damping ratio decreases firstly and, then, increases. Moreover, for a specific value of the amplitude, by increasing $\mu$, the damping ratio increases.

The investigations showed that, for $A>0.8$, the obtained solution in Eq. (50) has low accuracy. For instance, if the amplitude is selected as $A=1$, the following solution will be obtained by AGM:

$$
u(t)=1.02 e^{-1.5 t} \cos (7.5 t-0.1974) .
$$

The above solution, which is compared with the numerical solution in Figure 16, has low accuracy and, consequently, it should be improved in the same manner as the previous examples. According to Eq. (17),

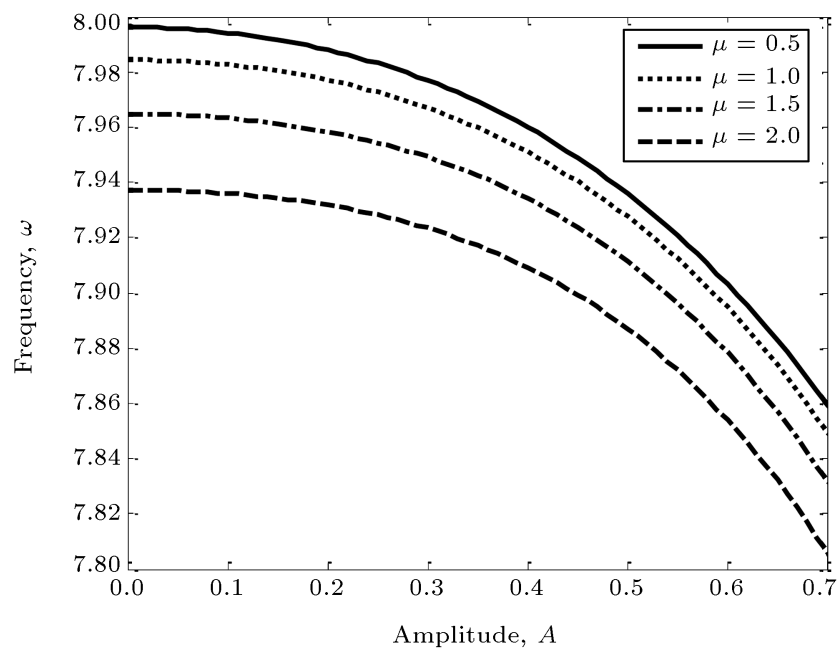

Figure 14. The variation of frequency with respect to the initial amplitude for Example 3.

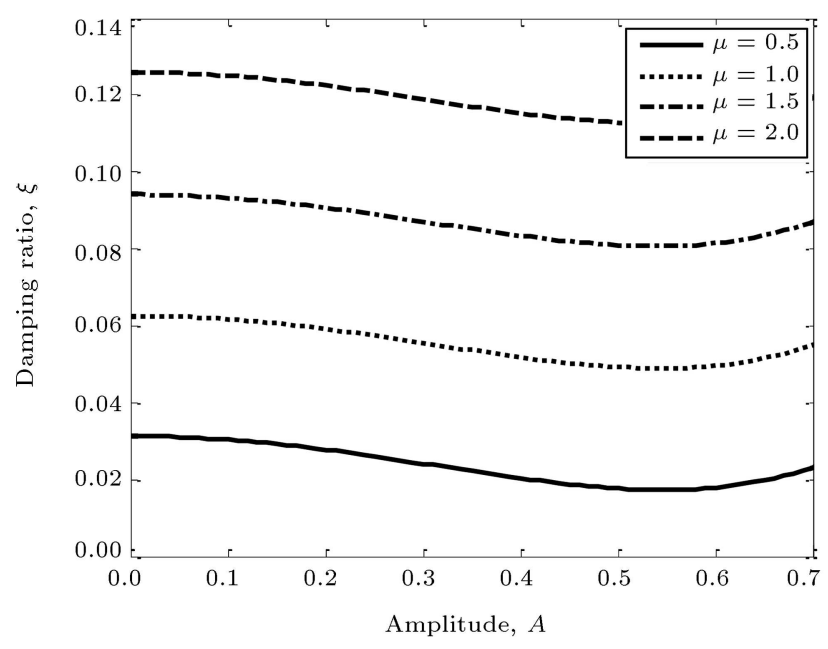

Figure 15. The variation of damping ratio with respect to the initial amplitude for Example 3. 
the Lagrange multiplier is obtained as follows:

$$
\lambda(\tau)=\frac{\sin (7.9373(\tau-t))}{7.9373} e^{(\tau-t)} .
$$

Considering Eq. (52) as the zero-order solution $\left(u_{0}(t)\right)$ and substituting it into Eq. (13), the improved solution $\left(u_{\text {IAGM }}\right)$ can be obtained through the following correction functional:

$$
\begin{aligned}
u_{\mathrm{IAGM}}(t)= & u_{0}(t)+\int_{0}^{t} \frac{\sin (7.9373(\tau-t))}{7.9373} e^{(\tau-t)}\left\{u_{0}^{\prime \prime}(\tau)\right. \\
& +\left(\mu-\eta u_{0}^{2}(\tau)+\gamma u_{0}^{4}(\tau)\right) u_{0}^{\prime}(\tau) \\
& \left.+\Omega^{2} u_{0}(\tau)-\beta u_{0}^{3}(\tau)-\varepsilon u_{0}^{5}(\tau)\right\} d \tau
\end{aligned}
$$

As is clear in Figures 16 and 17, AGM improved solution $\left(u_{\mathrm{IAGM}}\right)$ is in good agreement with the numerical results.

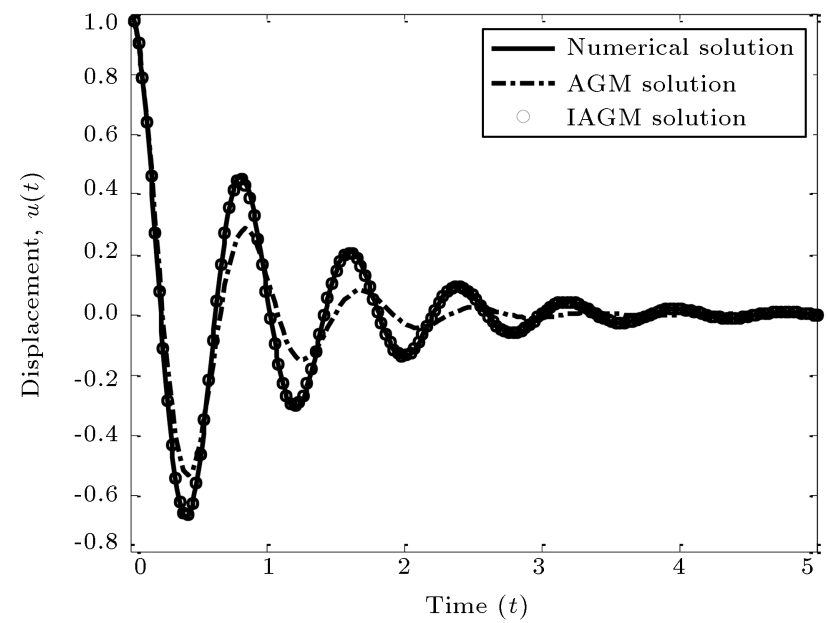

Figure 16. The solutions obtained by Akbari-Ganji's Method (AGM), Improved AGM (IAGM), and numerical method for Example 3, $A=1$.

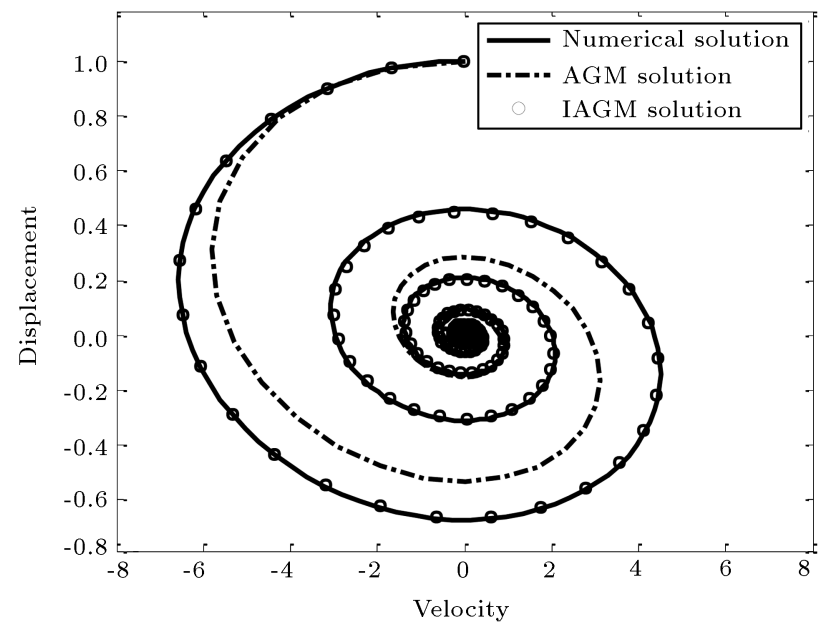

Figure 17. The phase plane diagram obtained by Akbari-Ganji's Method (AGM), Improved AGM (IAGM), and numerical method for Example $3, A=1$.

\section{Conclusion}

In this paper, Akbari-Ganji's Method (AGM) was employed for solving three damped oscillatory systems with linear and nonlinear terms in damping coefficients. Considering a simple trial solution for the corresponding differential equation and, then, by applying the initial conditions to the supposed answer, the main differential equation, and its derivatives, the constant coefficients of trial solution can be determined. AGM is a very simple procedure, because it can be easily implemented and does not need to introduce a new independent variable, which is common in some analytical techniques such as harmonic balance method. Although AGM produces accurate solutions, in some problems, lower accuracy is achieved. In such cases, in contrast to the previously published papers proposed by a trial solution with higher-order terms, it was suggested that the solution obtained by AGM could be improved through the variational iteration method. The comparison of the results and those obtained by the numerical method confirmed the correctness and usefulness of the approach. Hence, AGM can be extended to other strong nonlinear problems with or without damping terms and, if necessary, its accuracy can be easily improved.

\section{Nomenclature}

AGM Akbari-Ganji's Method

IAGM Improved AGM

VIM Variational Iteration Method

$\omega$

$\xi \quad$ Damping ratio

$u(t) \quad$ Displacement (m)

$\dot{u}(t) \quad$ Velocity $(\mathrm{m} / \mathrm{s})$

$\ddot{u}(t) \quad$ Acceleration $\left(\mathrm{m} / \mathrm{s}^{2}\right)$

$A \quad$ Initial amplitude (m)

$B \quad$ Initial velocity $(\mathrm{m} / \mathrm{s})$

IC Initial Condition

$L \quad$ Linear operator

$N \quad$ Nonlinear operator

Variational operator

Constant parameter

Constant parameter

Constant parameter

Constant parameter

Constant parameter

Constant parameter 


\section{References}

1. Afsharfard, A. and Farshidianfar, A. "Design of nonlinear impact dampers based on acoustic and damping behavior", International Journal of Mechanical Sciences, 65(1), pp. 125-133 (2012).

2. Leung, A.Y.T., Guo, Z., and Yang, H.X. "Residue harmonic balance analysis for the damped Duffing resonator driven by a van der Pol oscillator", International Journal of Mechanical Sciences, 63(1), pp. 59-65 (2012).

3. Mansoori Kermani, M. and Dehestani, M. "Solving the nonlinear equations for one-dimensional nanosized model including Rydberg and Varshni potentials and Casimir force using the decomposition method", Applied Mathematical Modelling, 37(5), pp. 3399-3406 (2013).

4. Zhang, W., Wang, F.-X., and Zu, J.W. "Local bifurcations and codimension- 3 degenerate bifurcations of a quintic nonlinear beam under parametric excitation", Chaos, Solitons \& Fractals, 24(4), pp. 977-998 (2005).

5. Mohammadian, M. and Shariati, M. "Approximate analytical solutions to a conservative oscillator using global residue harmonic balance method", Chinese Journal of Physics, 55(1), pp. 47-58 (2017).

6. Mohammadian, M. and Akbarzade, M. "Higher-order approximate analytical solutions to nonlinear oscillatory systems arising in engineering problems", Archive of Applied Mechanics, 87(8), pp. 1317-1332 (2017).

7. Mohammadian, M. "Application of the global residue harmonic balance method for obtaining higher-order approximate solutions of a conservative system", International Journal of Applied and Computational Mathematics, 3(3), pp. 2519-2532 (2017).

8. Mohammadian, M., Pourmehran, O., and Ju, P. "An iterative approach to obtaining the nonlinear frequency of a conservative oscillator with strong nonlinearities", International Applied Mechanics, 54(4), pp. 470-479 (2018).

9. Ganji, S.S., Ganji, D.D., Davodi, A.G., and Karimpour, S. "Analytical solution to nonlinear oscillation system of the motion of a rigid rod rocking back using max-min approach", Applied Mathematical Modelling, 34(9), pp. 2676-2684 (2010).

10. Yazdi, M.K., Ahmadian, H., Mirzabeigy, A., and Yildirim, A. "Dynamic analysis of vibrating systems with nonlinearities", Communications in Theoretical Physics, 57(2), pp. 183-187 (2012).

11. De Rosa, M.A. and Lippiello, M. "Nonlocal Timoshenko frequency analysis of single-walled carbon nanotube with attached mass: An alternative Hamiltonian approach", Composites Part B: Engineering, 111, pp. 409-418 (2017).
12. Yazdi, M.K., Mirzabeigy, A., and Abdollahi, H. "Nonlinear oscillators with non-polynomial and discontinuous elastic restoring forces", Nonlinear Science Letters A, 3(1), pp. 48-53 (2012).

13. Baltanás, J.P., Trueba, J.L., and Sanjuán, M.A.F. "Energy dissipation in a nonlinearly damped Duffing oscillator", Physica D: Nonlinear Phenomena, 159(12), pp. 22-34 (2001).

14. Liao, S.-J. "An analytic approximate technique for free oscillations of positively damped systems with algebraically decaying amplitude", International Journal of Non-Linear Mechanics, 38(8), pp. 1173-1183 (2003).

15. Cveticanin, L. "Oscillators with nonlinear elastic and damping forces", Computers \& Mathematics with Applications, 62(4), pp. 1745-1757 (2011).

16. Shamsul Alam, M., Roy, K.C., Rahman, M.S., and Mossaraf Hossain, M. "An analytical technique to find approximate solutions of nonlinear damped oscillatory systems", Journal of the Franklin Institute, 348(5), pp. 899-916 (2011).

17. Wu, B.S. and Sun, W.P. "Construction of approximate analytical solutions to strongly nonlinear damped oscillators", Archive of Applied Mechanics, 81(8), pp. 1017-1030 (2011).

18. Nourazar, S. and Mirzabeigy, A. "Approximate solution for nonlinear Duffing oscillator with damping effect using the modified differential transform method", Scientia Iranica, 20(2), pp. 364-368 (2013).

19. Cveticanin, L. "On the truly nonlinear oscillator with positive and negative damping", Applied Mathematics and Computation, 243, pp. 433-445 (2014).

20. Ene, R.D., Marinca, V., and Marinca, B. "Free oscillations of a nonlinear oscillator with an exponential nonviscous damping", Applied Mechanics and Materials, 801, pp. 38-42 (2015).

21. Herisanu, N. and Marinca, V. "An optimal homotopy asymptotic approach to a damped dynamical system of a rotating electrical machine", Applied Mechanics and Materials, 801, pp. 202-206 (2015).

22. Razzak, M.A. and Molla, M.H.U. "A new analytical technique for strongly nonlinear damped forced systems", Ain Shams Engineering Journal, 6(4), pp. 1225-1232 (2015).

23. Sheikholeslami, M. and Ganji, D.D. "Nanofluid flow and heat transfer between parallel plates considering Brownian motion using DTM", Computer Methods in Applied Mechanics and Engineering, 283, pp. 651-663 (2015).

24. Mohammadian, M., Hosseini, S.M., and Abolbashari, M.H. "Lateral vibrations of embedded hetero-junction carbon nanotubes based on the nonlocal strain gradient theory: Analytical and differential quadrature element (DQE) methods", Physica E: Low-dimensional Systems and Nanostructures, 105, pp. 68-82 (2018). 
25. Sheikholeslami, M. and Ganji, D.D. "Magnetohydrodynamic flow in a permeable channel filled with nanofluid", Scientia Iranica, B, 21(1), pp. 203-212 (2014).

26. Ebaid, A., Rach, R., and El-Zahar, E. "A new analytical solution of the hyperbolic Kepler equation using the Adomian decomposition method", Acta Astronautica, 138, pp. 1-9 (2017).

27. Pakdemirli, M. "Perturbation-iteration method for strongly nonlinear vibrations", Journal of Vibration and Control, 23(6), pp. 959-969 (2017).

28. Akbari, M.R., Ganji, D.D., Majidian, A., and Ahmadi, A.R. "Solving nonlinear differential equations of Vanderpol, Rayleigh and Duffing by AGM", Frontiers of Mechanical Engineering, 9(2), pp. 177-190 (2014).

29. Mirgolbabaee, H., Ledari, S.T., and Ganji, D.D. "New approach method for solving Duffing-type nonlinear oscillator", Alexandria Engineering Journal, 55(2), pp. 1695-1702 (2016).

30. Akbari, M.R., Ganji, D.D., Nimafar, M., and Ahmadi, A.R. "Significant progress in solution of nonlinear equations at displacement of structure and heat transfer extended surface by new AGM approach", Frontiers of Mechanical Engineering, 9(4), pp. 390-401 (2014).

31. Akbari, M.R., Ganji, D.D., Rostami, A.K., and Nimafar, M. "Solving nonlinear differential equation governing on the rigid beams on viscoelastic foundation by AGM", Journal of Marine Science and Application, 14(1), pp. 30-38 (2015).

32. Mirgolbabaee, H., Ledari, S.T., and Ganji, D.D. "An assessment of a semi analytical AG method for solving nonlinear oscillators", New Trends in Mathematical Sciences, 4(1), pp. 283-299 (2016).

33. He, J. "A new approach to nonlinear partial differential equations", Communications in Nonlinear Science and Numerical Simulation, 2(4), pp. 230-235 (1997).
34. Daeichi, M. and Ahmadian, M. "Application of variational iteration method to large vibration analysis of slenderness beams considering mid-plane stretching", Scientia Iranica. Transactions B, Mechanical Engineering, 22(5), pp. 1911-1917 (2015).

35. Mohammadian, M. "Application of the variational iteration method to nonlinear vibrations of nanobeams induced by the van der Waals force under different boundary conditions", The European Physical Journal Plus, 132(4), p. 169 (2017).

36. Witten, M. and Siegel, D. "A kinetics model of abrin binding in a virus transformed lymphocyte cell culture", Bulletin of Mathematical Biology, 44(4), pp. 453-476 (1982).

\section{Biographies}

Mostafa Mohammadian received his BSc degree in Mechanical Engineering from Ferdowsi University of Mashhad, Iran in 2007 and the MSc degree in applied Mechanical Engineering from Semnan University, Semnan, Iran in 2009. Also, he received his PhD degree in Mechanical Engineering from Ferdowsi University of Mashhad, Iran in 2019. Moreover, he is a faculty member and the Head of the Department of Mechanical Engineering in Islamic Azad University, Gorgan branch, Kordkuy center, Iran. His research interests include nonlinear dynamics and vibration, numerical methods, optimization and approximate analytical methods.

Mahmoud Shariati is a Full Professor in Ferdowsi University of Mashhad, Iran. He was educated in the mechanical engineering field and, now, is teaching at the Department of Mechanical Engineering in Ferdowsi University of Mashhad, Iran. His research focuses on the nonlinear dynamics, fracture mechanics and fatigue, stress analysis, design of mechanical structures, and nano-mechanics. He has published more than 250 scientific papers and books. 